\title{
Effectiveness of ustekinumab dose escalation in Crohn's disease patients with insufficient response to standard-dose subcutaneous maintenance therapy
}

Uri Kopylov $^{1}$ (D) | Jurij Hanzel ${ }^{3}$ | Claire Liefferinckx ${ }^{4}$ | Davide De Marco ${ }^{2}$ | Nicola Imperatore $^{5}$ (D) | Nikolas Plevris ${ }^{6}$ (D) | Iria Baston-Rey ${ }^{7}$ | Richard J. Harris ${ }^{8}$ | Marie Truyens $^{9}$ | Viktor Domislovic ${ }^{10}$ | Stephan Vavricka ${ }^{11}$ | Vince Biemans ${ }^{12,13}$ Sally Myers ${ }^{14}$ | Shaji Sebastian ${ }^{14}$ (D) | Shomron Ben-Horin ${ }^{1}$ | Yago González Lama ${ }^{15}$ | Cyrielle Gilletta $^{16}$ | Bar-Gil Shitrit Ariella ${ }^{17}$ | Zuzana Zelinkova ${ }^{18}$ | Roni Weishof ${ }^{19}$ | Darragh Storan $^{20}$ | Eran Zittan ${ }^{21}$ (D) | Klaudia Farkas ${ }^{22}$ | Tamas Molnar ${ }^{22}$ | Denis Franchimont $^{4}$ | Anneline Cremer ${ }^{4}$ | Waqqas Afif ${ }^{2}$ | Fabiana Castiglione ${ }^{5}$ | Charles Lees $^{6}$ | Manuel Barreiro-de Acosta ${ }^{7}$ | Triana Lobaton ${ }^{9}$ | Glen Doherty ${ }^{20}$ | Zeljko Krznaric $^{19}$ | Marieke Pierik ${ }^{12}$ | Frank Hoentjen ${ }^{13}$ | David Drobne ${ }^{3}$

\footnotetext{
${ }^{1}$ Tel Aviv, Israel

${ }^{2}$ Montreal, Canada

${ }^{3}$ Ljubljana, Slovenia

${ }^{4}$ Brussels, Belgium

${ }^{5}$ Naples, Italy

${ }^{6}$ Edinburgh, UK

${ }^{7}$ Santiago de Compostela, Spain

${ }^{8}$ Southampton, UK

${ }^{9}$ Ghent, Belgium

${ }^{10}$ Zagreb, Croatia

${ }^{11}$ Zurich, Switzerland

${ }^{12}$ Maastricht, The Netherlands

${ }^{13}$ Nijmegen, The Netherlands

${ }^{14}$ Hull, UK

${ }^{15}$ Madrid, Spain

${ }^{16}$ Toulouse, France

17 Jerusalem, Israel

${ }^{18}$ Bratislava, Slovakia

${ }^{19}$ Haifa, Israel

${ }^{20}$ Dublin, Ireland

${ }^{21}$ Afula, Israel

${ }^{22}$ Szeged, Hungary
}

\begin{abstract}
Summary
Background: Ustekinumab is effective in Crohn's disease. However, a substantial proportion of patients will not respond or lose response to ustekinumab. The current evidence to support the effectiveness of dose-optimisation for ustekinumab nonresponse is limited.

Aim: To assess the effectiveness of dose escalation of ustekinumab.

Methods: This was a multicentre retrospective cohort study. We included active Crohn's disease patients who received a standard-dose intravenous induction and at least one subcutaneous ustekinumab $90 \mathrm{mg}$ dose. All enrolled patients received dose escalation by either shortening the interval between the doses to every 4 or 6 weeks, intravenous reinduction or a combination of strategies. The primary outcome of the study was clinical response at week 16 after dose escalation.

Results: A total of 142 patients (22 centres/14 countries) were included. The patients were dose-escalated after a median treatment duration of 30 weeks. At week 16 from escalation, 73/142 (51.4\%) responded to treatment, including 55/142 (38.7\%) in clinical remission. Corticosteroid-free remission was achieved in 6/34 (17.6\%) patients on corticosteroids at the time of escalation; 118/142 (83\%) continued treatment beyond week 16. Follow-up data beyond week 16 were available for 74/118 (62.7\%) patients. On the last follow-up, 51/98 (52\%) patients with available data responded to treatment, including $41 / 98$ (42\%) in clinical remission.
\end{abstract}


Ramat-Gan and Sackler Medical School, Tel Aviv University, Israel. Email: ukopylov@gmail.com
Conclusions: Intensification of ustekinumab maintenance dosage was effective in over $50 \%$ of the patients. This strategy should be considered in patients who are nonresponsive to every 8 weeks ustekinumab maintenance dosing.

\section{1 | INTRODUCTION}

Ustekinumab (UST) is a monoclonal antibody that targets the p40 subunit of interleukin (IL) 12 and 23. UST is effective for treatment of Crohn's disease (CD) and ulcerative colitis. ${ }^{1-4}$ In addition to randomised controlled trials, multiple real-world studies corroborated the effectiveness of UST in the treatment of CD. ${ }^{5-19}$ However, the risk of primary and secondary loss of response to UST has not been extensively reported. In the recently published long-term extension of the UNITI trials, clinical remission was well maintained from week 44 to 92 for both every 12 weeks (q12w) (77.4\%/72.6\%) and every 8 weeks (q8w) regimen (84.1\%/74.4\%). ${ }^{3}$ Nevertheless, the rates of sustained response appear to be lower in the real-world setting. In a multicentre real-world study from the Netherlands, the probability of remaining on ustekinumab treatment at 52 weeks was $62.9 \%{ }^{7}$ Similar results were reported in a recent Belgian cohort study. ${ }^{13}$

The vast majority of $C D$ patients treated with ustekinumab in the real-world setting have failed at least two previous biologics. ${ }^{9}$ Additional therapeutic options in these patients are severely limited. Therefore, there is a need for an effective strategy for management of nonresponse to ustekinumab.

The standard ustekinumab treatment regimen for $C D$ includes an intravenous (IV) induction (adjusted $6 \mathrm{mg} / \mathrm{kg}$ dose) followed by UST subcutaneous (SC) 90 mg administered every 12 or 8 weeks. No significant differences between both dose regimens with regards to clinical efficacy were demonstrated in the UNITI trials. ${ }^{2,3}$ However, every 8 weeks regimen (q8w) was more effective in achieving endoscopic response. ${ }^{1}$ Dose escalation $q 8 \mathrm{w}$ in patients failing to respond to $\mathrm{q} 12 \mathrm{w}$ regimen has been shown to be effective. ${ }^{3,20}$ Yet, there are scarce data to support efficacy of further dose escalation in patients failing $q 8 w$ dosing. In a Groupe d'Étude Thérapeutique des Affections Inflammatoires du Tube Digestif (GETAID) cohort study, published as a conference report, clinical response was obtained in 43/69 (57.1\%) of the patients after 2.1 months post every 4 weeks (q4w) optimisation. ${ }^{21}$ In addition, successful intravenous reinduction was described in a case series of three patients with either primary or secondary nonresponse to UST. ${ }^{22}$

Additional data to support a dose-escalation regimen in CD patients failing to respond to ustekinumab are required. The aim of the present study was to evaluate the effectiveness and safety of dose escalation of UST in patients who did not respond or lost response to ustekinumab q8w maintenance dose.

\section{2 | METHODS}

This was a multicentre retrospective cohort study. The protocol was reviewed and approved by the Clinical committee of the European
Crohn's and Colitis organisation (ECCO).The cohort study included active (defined as Harvey-Bradshaw Index (HBI) $\geq 5$ or Crohn's disease activity index (CDAI) $\geq 150)$ CD patients. The included patients received at least two doses of ustekinumab (IV induction of $6 \mathrm{mg} / \mathrm{kg}$ followed by SC $90 \mathrm{mg}$ injection) and were either dose-escalated to q4w or q6w or, alternatively, received an IV reinduction $(6 \mathrm{mg} / \mathrm{kg})$ instead of a scheduled SC dose or a combination of IV and SC escalation. Patients without active disease as per $\mathrm{CDAl} / \mathrm{HBI}$, and patients with pouch or ostomy were excluded from the study. Patients who started q12w maintenance after IV induction could be included in the study only if they were escalated to $q 8 w$ regimen prior to further escalation.

The primary objective of the study was clinical response $(\Delta \mathrm{HBI} \geq 3$ or $\triangle \mathrm{CDAl} \geq 70$ ) at week 16 from the escalation. Secondary outcomes included clinical remission ( $\mathrm{HBI} \leq 4$; CDAI $\leq 150)$ at week 16; C-reactive protein (CRP) normalisation (as per cut-off levels used in the corresponding institutions) at week 16 (in patients with elevated CRP at the time of escalation); steroid-free clinical remission at week 16 (in patients receiving systemic corticosteroids at the time of escalation) and steroid-free clinical response at week 16. In addition to week 16 from the escalation, we addressed long-term outcomes (last visit after week 16, up to 52 weeks from dose escalation) when available. Last follow-up secondary outcomes included clinical response and remission, steroid-free response and remission, improvement in perianal fistula drainage or fistula healing (based on physician's assessment or per imaging where available). We also collected safety outcomes including patient-reported adverse events, serious adverse events (resulting in hospitalisation or treatment discontinuation), hospitalisations and CD-related surgery following dose escalation.

\section{3 | STATISTICAL METHODS}

Descriptive statistics were presented as means \pm standard deviations (SD) for parametric variables, medians with interquartile ranges (IQR) for nonparametric continuous variables and percentages for categorical variables. Categorical variables were analysed by chi-square/ Fisher's exact test and continuous variables by $t$ test/Mann Whitney test as appropriate. A two-tailed $P<0.05$ was considered statistically significant. We constructed a multivariate logistic regression model to identify the independent predictors of ustekinumab discontinuation at week 52. Variables with significance level $<0.1$ on the univariate analysis were included in the model. To investigate the effect of the variables on duration of continued UST treatment, we performed a survival analysis, Kaplan-Meier survival curve and Cox multivariate proportional hazard model. The model included variables with significance level $<0.1$ on univariate analysis. We used IBM SPSS statistic (Version 20.0) in performing the analysis. Ethical approval was obtained as per the requirements of each of the participating centres. 


\section{4 | RESULTS}

One hundred and forty-two patients from 22 centres in 14 countries (13 Europe and 1 Canada) were included in the study. The clinical and demographic characteristics of the patients are detailed in Table 1. Only $3.5 \%$ of the patients were biologic-naive, $23.2 \%$ received one prior biologic, $39.4 \%$ received two prior biologics, $32.4 \%$ received three prior biologics and $1.4 \%$ received four prior biologics. Fiftyseven (40\%) of the patients had previously received both anti-TNFs and vedolizumab. One hundred and six (77\%) had elevated CRP at dose escalation. All patients received intravenous UST induction and SC maintenance (90 mg SC q8w); 9/142 (6.3\%) received q12w and were escalated to $\mathrm{q} 8 \mathrm{w}$ before inclusion in the study. The mean duration of SC maintenance therapy before dose escalation was 29 (IQR 18-46) weeks with a median of four (IQR 2-6) injections. All patients had active CD (median HBI- 8 (IQR 6-12) at the time of dose escalation.

Ninety-one (64.1\%) of the patients were escalated to $q 4 \mathrm{w}$ regimen, 20/142 (14.1\%) patients were escalated to q6w, 14/142 (9.9\%) patients received an IV reinduction and 17/142 (12\%) a combination of IV reinduction and interval shortening.

\section{5 | TREATMENT OUTCOMES}

\section{1 | Week 16 from escalation}

Clinical response was achieved by $73 / 142$ (51.4\%) patients, including 55/142 (38.7\%) patients in clinical remission. CRP normalised in $21 / 98$ (21.4\%) patients with CRP values available at both time points. Systemic corticosteroids were discontinued in 23/34 (67.6\%) patients who were on corticosteroids at the time of escalation. Corticosteroid-free remission was achieved by 6/34(17.6\%) patients on corticosteroids upon escalation. Corticosteroid treatment was started by $6 / 108$ (5.6\%) patients who were not treated with corticosteroids upon dose escalation.

The likelihood of achieving clinical remission was similar between patients who received intravenous reinduction vs those who received SC interval shortening (Table 2). None of the clinical or demographic parameters were associated with likelihood of response (Table 2). Ustekinumab was discontinued by 24 (17\%) of the patients due to clinical nonresponse (17/91 (18.7\%) patients who escalated to $\mathrm{q} 4 \mathrm{w}, 1 / 20$ (5\%) patients escalated to q6w, 5/14 (35.6\%) patients who received IV reinduction and $1 / 17(5.9 \%)$ patients who received both IV reinduction and SC escalation respectively).

\section{2 | Maintenance}

Clinical follow-up beyond week 16 was available for 74/118 (62.7\%) patients who continued treatment (median duration of follow-up was 26 (IQR 32-52) weeks); 44 patients did not have follow-up data available beyond week 16 . Maintenance
TABLE 1 Clinical and demographic characteristics of the included patients

\begin{tabular}{|c|c|}
\hline $\begin{array}{l}\text { Age at treatment onset, years (median, interquartile } \\
\text { range) }\end{array}$ & $35(26-49)$ \\
\hline Disease duration (median, interquartile range) & $10(5-17)$ \\
\hline \multicolumn{2}{|l|}{ Gender (n, \%) } \\
\hline Male & $55(38.7 \%)$ \\
\hline Female & $87(61.3 \%)$ \\
\hline \multicolumn{2}{|l|}{ Behaviour (n, \%) } \\
\hline Nonstricturing nonpenetrating & $51(35.9 \%)$ \\
\hline Stricturing & $53(37.3 \%)$ \\
\hline Penetrating & $38(26.8 \%)$ \\
\hline \multicolumn{2}{|l|}{ Smoking (n, \%) } \\
\hline Never & $101(70.9 \%)$ \\
\hline Active smoker & $20(14.2 \%)$ \\
\hline Past smoker & $21(14.9 \%)$ \\
\hline \multicolumn{2}{|l|}{ CD location (n, \%) } \\
\hline Small bowel & 25 (17.6\%) \\
\hline Colon & $31(21.8 \%)$ \\
\hline Small bowel and colon & $86(60.6 \%)$ \\
\hline History of perianal disease (n, \%) & $56(40.1 \%)$ \\
\hline History of previous abdominal surgery (n, \%) & $81(57 \%)$ \\
\hline Active perianal disease at escalation (n, \%) & $33(23.2 \%)$ \\
\hline \multicolumn{2}{|l|}{ Number of previous biologic therapies (n, \%) } \\
\hline \multicolumn{2}{|l|}{ Previous biologics (n, \%) } \\
\hline None & $5(3.5 \%)$ \\
\hline 1 & $33(23.2 \%)$ \\
\hline 2 & $56(39.4 \%)$ \\
\hline 3 & $46(32.4 \%)$ \\
\hline 4 & $2(1.4 \%)$ \\
\hline Elevated CRP (n, \%) & $106(77.4 \%)$ \\
\hline Systemic corticosteroids at escalation (n, \%) & $34(24 \%)$ \\
\hline Concomitant immunomodulators at escalation (n, \%) & 24 (16.9) \\
\hline
\end{tabular}

Abbreviations: CD, Crohn's disease; CRP, C-reactive protein.

outcomes were reported for patients who had available data beyond week 16 or discontinued treatment by week $16(n=98)$ (Figures 1 and 2). At last follow-up, 51/98 (52\%) patients responded to treatment, including 41/98 (42\%) who achieved clinical remission (with the exclusion of patients who discontinued ustekinumab by week 16, 51/74 (68.9\%) responded including $41 / 74$ (55.4\%) who achieved clinical remission). Eleven of $53(20.7 \%)$ patients who did not respond at week 16 and had available maintenance data responded subsequently, including 9/54 (17\%) who achieved clinical remission. Corticosteroid-free remission was achieved by $9 / 34$ (26.5\%) patients who were treated with corticosteroids at dose escalation (including six patients who had achieved corticosteroid-free remission by week 16 as described above). Corticosteroids were initiated in two additional patients after week 16; overall, 8/108 (7.4\%) 


\begin{tabular}{|c|c|c|c|c|c|}
\hline & \multicolumn{4}{|c|}{ Week 16 response } & \multirow[b]{3}{*}{$P$} \\
\hline & \multicolumn{2}{|c|}{ no $(n=69)$} & \multicolumn{2}{|c|}{ yes $(n=73)$} & \\
\hline & $\mathrm{n}$ & $\%$ & $\mathrm{n}$ & $\%$ & \\
\hline $\begin{array}{l}\text { Age at treatment onset, years (median } \\
\text { interquartile range) }\end{array}$ & 34 & $(25-44)$ & 37 & $(27-50)$ & 0.25 \\
\hline \multicolumn{6}{|l|}{ Gender (n, \%) } \\
\hline Male & 22 & $31.9 \%$ & 33 & $45.2 \%$ & \multirow[t]{2}{*}{0.1} \\
\hline Female & 47 & $68.1 \%$ & 40 & $54.8 \%$ & \\
\hline \multicolumn{6}{|l|}{ CD location (n, \%) } \\
\hline Small bowel & 12 & $17.4 \%$ & 13 & $17.8 \%$ & \multirow[t]{3}{*}{0.99} \\
\hline Colon & 15 & $21.7 \%$ & 16 & $21.9 \%$ & \\
\hline Small bowel and colon & 42 & $60.9 \%$ & 44 & $60.3 \%$ & \\
\hline \multicolumn{6}{|l|}{ Behaviour (n, \%) } \\
\hline Nonstricturing nonpenetrating & 24 & $34.8 \%$ & 27 & $37.0 \%$ & \multirow[t]{3}{*}{0.2} \\
\hline Stricturing & 22 & $31.9 \%$ & 31 & $42.5 \%$ & \\
\hline Penetrating & 23 & $33.3 \%$ & 15 & $20.5 \%$ & \\
\hline \multicolumn{6}{|l|}{ Smoking (n, \%) } \\
\hline Never & 51 & $75.0 \%$ & 49 & $67.1 \%$ & \multirow[t]{2}{*}{0.5} \\
\hline Active smoker & 9 & $13.2 \%$ & 11 & $15.1 \%$ & \\
\hline \multicolumn{6}{|l|}{ Type of escalation } \\
\hline Past smoker & 8 & $11.8 \%$ & 13 & $17.8 \%$ & \multirow{5}{*}{0.48} \\
\hline Q4w & 43 & $62.3 \%$ & 48 & $65.8 \%$ & \\
\hline Q4w + IV reinduction & 6 & $8.7 \%$ & 10 & $13.7 \%$ & \\
\hline Q6w & 11 & $15.9 \%$ & 10 & $13.7 \%$ & \\
\hline IV reinduction & 9 & $13.0 \%$ & 5 & $6.8 \%$ & \\
\hline Elevated CRP (n, \%) & 56 & $82.1 \%$ & 52 & $72.9 \%$ & 0.2 \\
\hline $\begin{array}{l}\text { Systemic corticosteroids at escalation } \\
(\mathrm{n}, \%)\end{array}$ & 20 & $29.0 \%$ & 15 & $20.5 \%$ & 0.7 \\
\hline $\begin{array}{l}\text { Concomitant immunomodulators at } \\
\text { escalation ( } \mathrm{n}, \%)\end{array}$ & 12 & $17.4 \%$ & 12 & $16.4 \%$ & 0.9 \\
\hline
\end{tabular}

TABLE 2 Variables associated with week 16 response

Abbreviations: CD, Crohn's disease; CRP, C-reactive protein; IV, intravenous; Q4w- every 4 weeks; Q6w, every 6 weeks.

patients who were not on corticosteroids upon dose escalation were started on corticosteroids throughout the duration of the follow-up. Sixty-seven (68.4\%) patients continued ustekinumab treatment at last follow-up.

Kaplan-Meier curve for discontinuation-free survival appears on Figure 3. None of the clinical or demographic parameters, including the escalation strategy, was significantly associated with the duration of drug discontinuation-free survival (data not shown).

Endoscopic evaluation both before (within 4 weeks) escalation and after escalation (24 (IQR 16-32) weeks from escalation) was available for 23 patients. Mucosal healing was reported for $2 / 23$ (8.6\%) patients. An endoscopic improvement was reported for 11 (47.8\%) of 23 patients and no improvement for 10 (43.4\%) of 23 patients.

Of 33 patients with active perianal disease, improvement was reported by 14 (42.4\%) patients at week 16 . Out of 17 patients with active perianal disease (and available data) beyond week 16, improvement was reported by 11 (64.7\%) patients.

\section{6 | SAFETY}

Adverse events followed escalation were reported by 11 (7.7\%) of the 142 patient and included skin eruptions (two patients), upper respiratory tract infection that required hospitalisation and subsequent treatment discontinuation (one patient), acute gastroenteritis of unknown aetiology (two patients), clostridium difficile infection (one patient), CMV colitis (one patient), concentration disturbance (one patient), benign breast lump (one patient), cervical intraepithelial neoplasia grade 1 (one patient) and nonmelanoma skin cancer (one patient). Ten (7\%) patients required CD-related surgery (nine bowel resection and one perianal abscess drainage). 


\section{7 | DISCUSSION}

The results of our study suggest that CD patients who are nonresponsive to standard maintenance dosing of ustekinumab may benefit from dose escalation. Dose escalation was effective in about $50 \%$ of the patients, with most of the responders achieving clinical remission.

Primary and secondary nonresponse and treatment discontinuation are frequent for all treatments in IBD, including ustekinumab, with rates of treatment discontinuation approaching $40 \%{ }^{7,13}$ These high rates of nonresponse are not surprising considering the refractoriness of the patients included in the real-life cohorts published so far. ${ }^{9}$

To date, the strategy for management of loss of response to UST has not been clearly established. While for patients treated with tumour necrosis factor (TNF)-alpha inhibitors, therapeutic

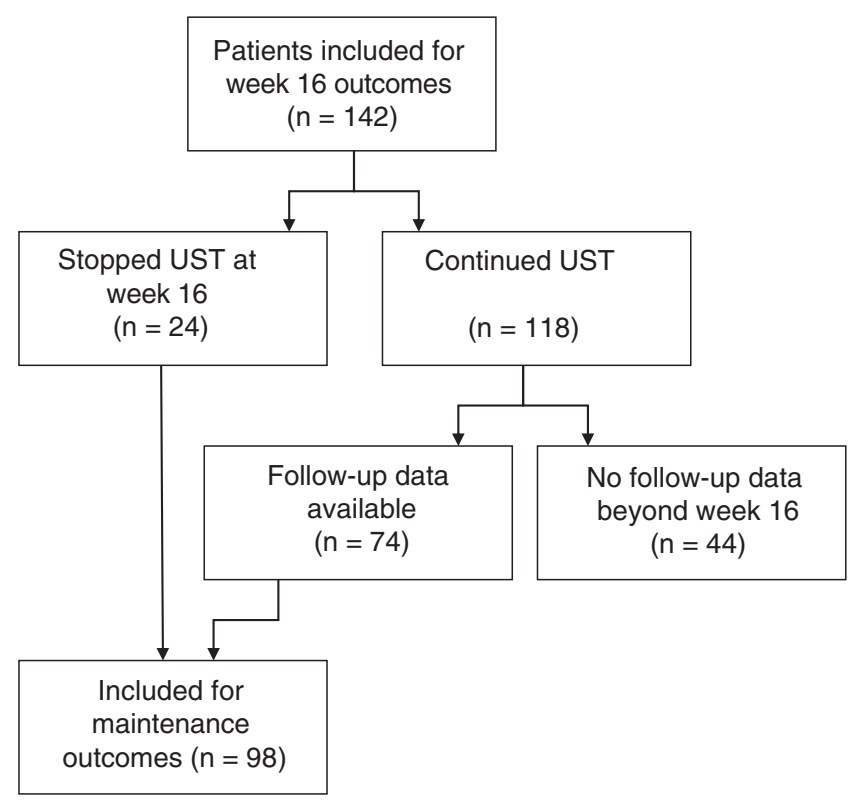

FIGURE 1 Patient inclusion chart. UST-ustekinumab drug monitoring may provide important guidance for selection of strategy. ${ }^{23-26}$ The correlation of ustekinumab levels with treatment outcomes is not as robust. ${ }^{6,12,27}$ Thus, the escalation strategy is currently empiric and nonstandardised.

Even though in the original UNITI 1/UNITI 2 trial no significant difference in the efficacy of both maintenance regimens was detected, ${ }^{2}$ the endoscopic response was more frequent in the $q 8 \mathrm{w}$ maintenance arm. ${ }^{1}$ In the long-term extension study of UNITI, patients who required dose-adjustment from $q 12 \mathrm{w}$ to $\mathrm{q} 8 \mathrm{w}$ regimen were less likely to remain in clinical remission throughout the duration of follow-up. ${ }^{3,20}$ However, only a few of the recent real-world studies included a significant proportion of patients who had received the currently approved intravenous induction regimen followed by a standard q12w or q8w maintenance, ${ }^{7,13,14}$ as most of these studies were published before the regulatory approval of intravenous ustekinumab for Crohn's disease. The effectiveness of further dose escalation in patients who did not respond to q8w dosing has not been reported so far, with the exception of the GETAID experience reported as an abstract (57\% response in 69 patients after a median of 2.1 months) ${ }^{21}$ and a very limited number of patients in early real-world series. ${ }^{12,16}$ In addition to interval shortening, a strategy of intravenous reinduction is utilised in some centres; however, the evidence to support it is minimal to date. ${ }^{28}$

Our study has some limitations. Primarily, this was a multicentre retrospective study that shares common limitations with similar efforts, namely heterogeneity in treatment strategies, lack of predefined timing of visits and missing biological data. Endoscopic response was not systematically evaluated, and perianal disease reporting was subjective and not universally available. Moreover, in many patients, the escalation was attempted relatively early on, and it is possible that some of the patients could have gradually improved with standard management strategy. As mentioned, the current study was descriptive and did not allow us to compare the effectiveness of the escalation to continuation of standard maintenance dosing. Our patients were all included by university-affiliated tertiary centres; nonetheless, it is possible that additional visits to community physicians could have occurred, leading to potentially missing prescription data or dose changes. Finally, we could not

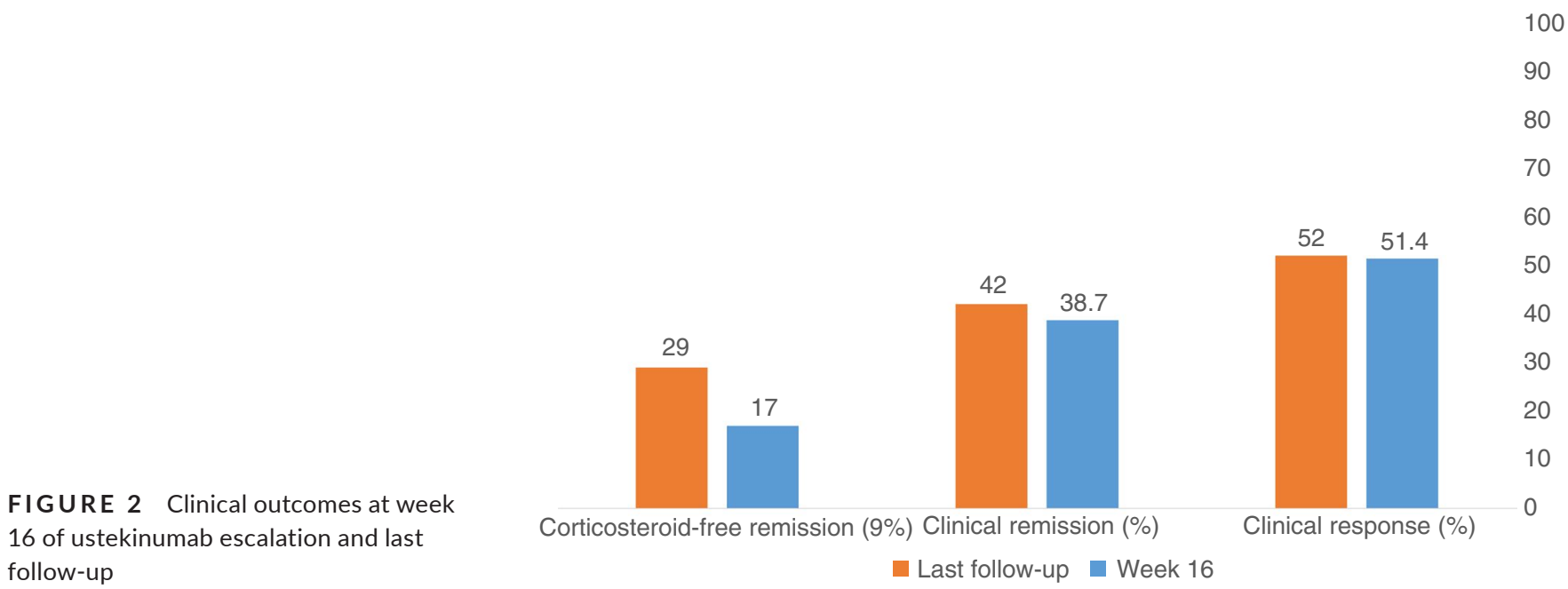




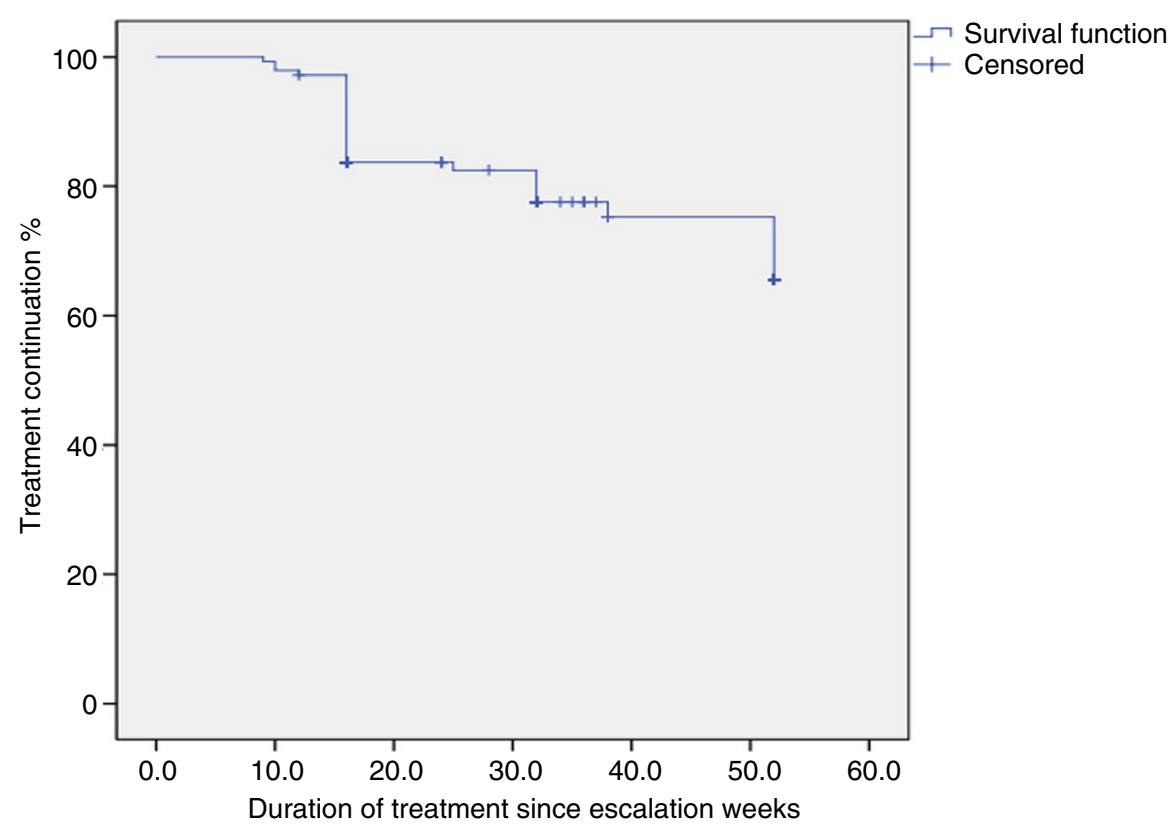

FIGURE 3 Kaplan-Meier curve of treatment discontinuation-free analysis detect differences in efficacy between the escalation strategies, albeit the study was not powered to detect such difference.

On the other hand, this study applied stringent inclusion criteria and clear treatment outcomes (only patients with available clinical scores were included). In addition, this was a large multinational and multicentre report that maximised the amount of the available data for analysis and reflected real-life practices of IBD specialists across the ECCO community. Finally, our study did not reveal any new safety concerns; however, a retrospective study is not designed to capture mild adverse events that could have resolved without being reported to the IBD specialist.

In summary, the data accumulated in our study support the efficacy of dose escalation in CD patients who do not respond or lost response to $\mathrm{q} 8 \mathrm{w}$ maintenance regimen. Our results merit validation in future prospective studies.

\section{ACKNOWLEDGEMENTS}

Uri Kopylov, designed the study, collected the data, performed the analysis and drafted the manuscriptJurij Hanzel, Claire Liefferinckx Davide De Marco, Nicola Imperatore, Nikolas Plevris, Iria BastonRey, Richard J Harris, Marie Truyens, Viktor Domislovic, Stephan Vavricka, Vince Biemans, Sally Myers, Shaji Sebastian, Shomron Ben- Horin, Yago González Lama, Cyrielle Gilletta, Bar-Gil Shitrit Ariella, Zuzana Zelinkova, Roni Weishof, Darragh Storan, Eran Zittan, Klaudia Farkas, Tamas Molnar, Denis Franchimont, Anneline Cremer, Waqqas Afif, Fabiana Castiglione, Charles Lees, Manuel Barreiro-de Acosta, Triana Lobaton, Glen Doherty, Zeljko Krznaric, Marieke Pierik, Frank Hoentjen, David Drobne, collected the data, reviewed the manuscript and contributed valuable scientific content.

Declaration of personal interests: Uri Kopylov received consultancy and speaker fees from Abbvie, Janssen, Takeda, MSD and Medtronic, and research grants from Janssen Takeda Medtronic. Jurij Hanzel received lecture fees from Biogen, Janssen and Takeda outside the submitted work. Claire Liefferinckx received consultancy fees from Takeda, and speaker fees from Sandoz, Janssen, Abbvie. Anneline Cremer received consultancy fees from Takeda and Janssen, and speaker fees from Pfizer and Abbvie. Denis Franchimont received educational grants from Abbvie, Takeda, MSD, fees or consultancy fees from Ferring, Falk, Chiesi, Abbvie, MSD, Centocor, Pfizer, Amgen, Janssen, Mundipharma and Hospira. Zeljko Krznaric received speaker fees from Abbvie, Takeda, MSD, Janssen, Oktal Pharma, Fresenius, Mylan and Pfizer. Manuel Barreiro-de Acosta has served as a speaker, a consultant and advisory member for or received research funding from MSD, Abbvie, Celltrion, Takeda, Janssen, Pfizer, Ferring, Faes Farma, Shire Pharmaceuticals, Dr Falk Pharma, Chiesi, Gebro Pharma, Roche, Otsuka Pharmaceuticals and Tillotts Pharma. Shomron Ben-Horin received consulting and advisory board fees and research support from AbbVie, Janssen, Takeda and Celltrion, and consulting and speaker fees from Pfizer, GlaxoSmithKline and MSD. Frank Hoentjen has served on advisory boards or as speaker or consultant for Abbvie, Celgene, JanssenCilag, MSD, Takeda, Celltrion, Teva, Sandoz and Dr Falk, and has received unrestricted grants from Dr Falk, Janssen-Cilag, Abbvie. Klaudia Farkas received speaker fees from AbbVie, Takeda, Janssen and Ferring. Tamás Molnár received speaker fees from MSD, AbbVie, Egis, Goodwill Pharma, Takeda, Pfizer and Teva. Eran Zittan received speaker fees, or research support or consulting fees from Janssen, Abbvie, Takeda, Neopharm and Pfizer. Nikolas Plevris received speaker fees and/or travel support from AbbVie, Takeda, Janssen, Norgine and Ferring. David Drobne has served as a speaker, a consultant and an advisory board member for MSD, Abbvie, Takeda, Pfizer, Janssen and Krka. Fabiana Castiglione received lecture fees from Abbvie, Takeda, Janssen, Sofar, Ferring. Richard James Harris received personal fees from Takeda, AbbVie and Janssen, and nonfinancial support from Falk. Yago González-Lama received consultancy, speaker fees and unrestricted grants from AbbVie, Janssen, Takeda, Pfizer, Ferring, Amgen, MSD. Shaji Sebastian holds research 
grants from Takeda, AbbVie, Warner Chilcott, Ferring, MSD, Biohit and Celgene, serves on the advisory boards of Janssen, Takeda, AbbVie, Merck, Ferring, Pharmacosmos, Warner Chilcott, Janssen, Falk Pharma, Biohit, TriGenix, Celgene and Tillotts Pharma, and has received speaker fees from Abbvie, Jansen, Merck, Warner Chilcott and Falk Pharma. Ariella Bar-Gil Shitrit- Grants; Takeda, Janssen. Lectures fee and advisory consultancy: Takeda, Janssen, Neopharm, Abbvie, Pfizer. Waqqas Afif has been a speaker advisory board member, and or clinical investigator for Abbvie, Arena Pharmaceuticals, Eli-Lilly, Janssen, Merck, Novartis, Pfizer, Prometheus, Takeda, Janssen, Merck, Warner Chilcott and Falk Pharma. Ariella Bar-Gil Shitrit- Grants; Takeda, Janssen. Lectures fee and advisory consultancy: Takeda, Janssen, Neopharm, Abbvie, Pfizer. Stephan Vavricka received consultancy and speaker fees from Abbvie, Falk, Ferring, Janssen, MSD, Pfizer, Takeda, UCB, Vifor. Glen Doherty has received educational and research grants, and/or professional fees from Abbvie, MSD, Janssen, Takeda/Shire, Tillott's, Dr Falk, Mylan, Biogen, Celltrion and Amgen. Dr Charlie Lees has received research support from Abbvie and Gilead, acted as a consultant to Abbvie, Janssen, Takeda, Pfizer, MSD, Hospira, Pharmacosmos, GSK, Gilead, Topivert, Vifor Pharma and Dr Falk and received speaking fees and travel support from Pfizer, Abbvie, MSD, Takeda, Shire, Ferring, Hospira, Warner-Chilcott and Dr Falk.Davide De Marco, Nicola Imperatore, , Iria Baston-Rey, Marie Truyens, Viktor DomislovicVince Biemans , Sally Myers, Cyrielle Gilletta, , Darragh Storan- did not have any conflict of interest to discloseAll authors approved the final version of the manuscriptNo funding was obtained for the study. No funding was obtained for the data analysis or writing of the paper.

\section{AUTHORSHIP}

Guarantor of the article: Uri Kopylov.

Author contributions: All authors collected the data and reviewed the manuscript. UK designed the study and drafted the manuscript. All authors reviewed and approved the final version of the manuscript.

\section{ORCID}

Uri Kopylov (iD https://orcid.org/0000-0002-7156-0588 Nicola Imperatore (iD https://orcid.org/0000-0003-3230-6832 Nikolas Plevris iD https://orcid.org/0000-0002-3229-8759 Vince Biemans (iD https://orcid.org/0000-0002-1361-8868 Shaji Sebastian (iD https://orcid.org/0000-0002-3670-6545 Eran Zittan iD https://orcid.org/0000-0003-3378-4932

\section{REFERENCES}

1. Rutgeerts P, Gasink C, Chan D, et al. Efficacy of ustekinumab for inducing endoscopic healing in patients with Crohn's disease. Gastroenterology. 2018;155:1045-1058.

2. Sandborn WJ, Gasink C, Gao L-L, et al. Ustekinumab induction and maintenance therapy in refractory Crohn's disease. N Engl J Med. 2012;367:1519-1528.

3. Sandborn WJ, Rutgeerts P, Gasink C, et al. Long-term efficacy and safety of ustekinumab for Crohn's disease through the second year of therapy. Aliment Pharmacol Ther. 2018;48:65-77.
4. Sands BE, Sandborn WJ, Panaccione R, et al. Ustekinumab as induction and maintenance therapy for ulcerative colitis. N Engl J Med. 2019;381:1201-1214.

5. Ahmed Z, Venkata K, Zhang N, et al. Comparative effectiveness of ustekinumab versus adalimumab in induction of clinical response and remission in Crohn's disease: experience of a real-world cohort at a tertiary care inflammatory bowel disease referral center. Gastroenterology Res. 2019;12:245-251.

6. Battat R, Kopylov U, Bessissow T, et al. Association between ustekinumab trough concentrations and clinical, biomarker, and endoscopic outcomes in patients with Crohn's disease. Clin Gastroenterol Hepatol. 2017;15:1427-1434.e2.

7. Biemans VBC, van der Meulen - de Jong AE, van der Woude CJ, et al. Ustekinumab for Crohn's disease: results of the ICC registry, a nationwide prospective observational cohort study. J Crohns Colitis. 2020;14:33-45.

8. Dayan JR, Dolinger M, Benkov K, et al. Real world experience with ustekinumab in children and young adults at a tertiary care pediatric inflammatory bowel disease center. J Pediatr Gastroenterol Nutr. 2019;69:61-67.

9. Engel T, Yung DE, Ma C, et al. Effectiveness and safety of Ustekinumab for Crohn's disease; systematic review and pooled analysis of real-world evidence. Dig Liver Dis. 2019;51:1232-1240.

10. Harris KA, Horst S, Gadani A, et al. Patients with refractory Crohn's disease successfully treated with ustekinumab. Inflamm Bowel Dis. 2016;22:397-401.

11. Iborra M, Beltrán B, Fernández-Clotet $A$, et al. Real-world shortterm effectiveness of ustekinumab in 305 patients with Crohn's disease: results from the ENEIDA registry. Aliment Pharmacol Ther. 2019;50:278-288.

12. Kopylov U, Afif W, Cohen A, et al. Subcutaneous ustekinumab for the treatment of anti-TNF resistant Crohn's disease-the McGill experience. J Crohns Colitis. 2014;8:1516-1522.

13. Liefferinckx C, Verstockt B, Gils A, et al. Long-term clinical effectiveness of ustekinumab in patients with Crohn's disease who failed biologic therapies: a national cohort study. J Crohns Colitis. 2019;13:1401-1409.

14. Verstockt B, Dreesen E, Noman M, et al. Ustekinumab exposure-outcome analysis in Crohn's disease only in part explains limited endoscopic remission rates. J Crohns Colitis. 2019;13:864-872.

15. Wils $P$, Bouhnik $Y$, Michetti $P$, et al. Subcutaneous ustekinumab provides clinical benefit for two-thirds of patients with Crohn's disease refractory to anti-tumor necrosis factor agents. Clin Gastroenterol Hepatol. 2016;14:242-250.e2.

16. Khorrami S, Ginard D, Marin-Jimenez I, et al. Ustekinumab for the treatment of refractory Crohn's disease: the Spanish experience in a large multicentre open-label cohort. Inflamm Bowel Dis. 2016;22:1662-1669.

17. Eberl A, Hallinen $T$, af Björkesten $C-G$, et al. Ustekinumab for Crohn's disease: a nationwide real-life cohort study from Finland (FINUSTE). Scand J Gastroenterol. 2019;54:718-725.

18. Harris RJ, McDonnell M, Young D, et al. Early real-world effectiveness of ustekinumab for Crohn's disease. Frontline Gastroenterology. 2019;11(2):111-116.

19. Bar-Gil Shitrit A, Ben-Ya'acov A, Siterman M, et al. Safety and effectiveness of ustekinumab for induction of remission in patients with Crohn's disease: a multicenter Israeli study. United Eur Gastroenterol J; 2050640620902956. [Epub ahead of print]. https://doi. org/10.1177/2050640620902956

20. Hanauer SB, Sandborn WJ, Feagan BG, et al. IM-UNITI: three-year efficacy, safety, and immunogenicity of ustekinumab treatment of Crohn's disease. J Crohns Colitis. 2020;14:23-32.

21. Fumery M, Peyrin-biroulet L, Nancey S, et al. OP24 Effectiveness and safety of ustekinumab 90 mg every 4 weeks in Crohn's disease. J Crohns Colitis. 2019;13:S016-S016. 
22. Park S, Evans E, Sandborn WJ, et al. Ustekinumab IV 6 mg/kg loading dose re-induction improves clinical and endoscopic response in Crohn's disease: a case series. Am J Gastroenterol. 2018;113:627-629.

23. Kopylov $U$, Mazor $\mathrm{Y}$, Yavzori M, et al. Clinical utility of antihuman lambda chain-based enzyme-linked immunosorbent assay (ELISA) versus double antigen ELISA for the detection of anti-infliximab antibodies. Inflamm Bowel Dis. 2012;18:1628-1633.

24. Ben-Horin S, Chowers Y. Tailoring anti-TNF therapy in IBD: drug levels and disease activity. Nat Rev Gastroenterol Hepatol. 2014;11:243-255.

25. Ungar B, Chowers Y, Yavzori M, et al. The temporal evolution of antidrug antibodies in patients with inflammatory bowel disease treated with infliximab. Gut. 2014;63:1258-1264.

26. Ungar B, Levy I, Yavne Y, et al. Optimizing anti-TNF-alpha therapy: serum levels of infliximab and adalimumab are associated with mucosal healing in patients with inflammatory bowel diseases. Clin Gastroenterol Hepatol. 2016;14:550-557. e2.

27. Adedokun OJ, Xu Z, Marano C, et al. Ustekinumab pharmacokinetics and exposure response in a phase 3 randomized trial of patients with ulcerative colitis: ustekinumab PK and exposure-response in UC. Clin Gastroenterol Hepatol. 2019. pii: S1542-3565(19)31403-X. [Epub ahead of print]. https://doi.org/10.1016/j.cgh.2019.11.059

28. Vazquez Moron JM, Rodriguez Moncada R, Pallares $\mathrm{MH}$. Intravenous ustekinumab reinduction as a Crohn's disease rescue strategy following a secondary non-response. Rev Esp Enferm Dig. 2019;111:721.

How to cite this article: Kopylov U, Hanzel J, Liefferinckx C, et al. Effectiveness of ustekinumab dose escalation in Crohn's disease patients with insufficient response to standard-dose subcutaneous maintenance therapy. Aliment Pharmacol Ther. 2020;00:1-8. https://doi.org/10.1111/apt.15784

\section{APPENDIX}

\section{Authors's complete affiliations}

Uri Kopylov: Department of Gastroenterology, Sheba Medical Center, Ramat Gan and Sackler Medical School, Tel Aviv University, Israel and Division of Gastroenterology, McGill University Health Center, Montreal, Quebec, Canada. Jurij Hanzel: Department of Gastroenterology, University Medical Centre Ljubljana, Ljubljana, Slovenia. Claire Liefferinckx, Denis Franchimont and Anneline Cremer: Department of Gastroenterology, Hôpital Erasme, ULB, Brussels, Belgium. Davide De Marco: Division of Gastroenterology, McGill University Health Center, Montreal, Quebec, Canada. Nicola Imperatore: Gastroenterology, Department of Clinical Medicine and Surgery, School of Medicine Federico II of Naples, Italy; Nikolas Plevris: The Edinburgh IBD Unit, Western General Hospital, Edinburgh, UK. Iria Baston-Rey: Unidad Ell, Servicio de Aparato Digestivo, Hospital Clínico Universitario de Santiago de Compostela, Santiago de Compostela, A Coruña, España. Richard J Harris: Department of Gastroenterology, University Hospital Southampton NHS Foundation Trust, Southampton, UK. Marie Truyens: Department of Gastroenterology, Ghent University
Hospital, Ghent, Belgium. Viktor Domislovic: Department of Gastroenterology and Hepatology, University Hospital Centre Zagreb, Croatia. Stephan Vavricka: Center for Gastroenterology and Hepatology, Zurich, Switzerland. Vince Biemans: Department of Internal Medicine, Division of Gastroenterology and Hepatology, Maastricht University Medical Center+, Maastricht, Netherlands and Department of Gastroenterology and Hepatology, Radboud University Medical Center, Nijmegen, the Netherlands. Sally Myers and Shaji Sebastian: IBD unit, Hull University Teaching Hospitals NHS Trust, Hull, UK. Shomron BenHorin: Department of Gastroenterology, Sheba Medical Center, Ramat Gan and Sackler Medical School, Tel Aviv University, Israel. Yago González Lama: IBD Unit,/ Gastroenterology and Hepatology Dpt, Hospital Universitario Puerta de Hierro, Madrid, Spain. Cyrielle Gilletta: Service de Gastroentérologie et Nutrition, CHU Toulouse Rangueil, France. Bar-Gil Shitrit Ariella: IBD MOM Unit, Digestive Diseases Institute, Shaare Zedek Medical Center, Affiliated with the Medical School, Hebrew University, Jerusalem, Israel. Zuzana Zelinkova: Department of Gastroenterology and Gastrointestinal Endoscopy, St Michael's Hospital, Bratislava, Slovakia. Roni Weishof: Gastroenterology Department, Rambam Health Care Campus, Haifa, Israel and Bruce Rappaport School of Medicine, Technion-Israel Institute of Technology, Haifa, Israel. Darragh Storan: Centre for Colorectal Disease, St. Vincent's University Hospital and School of Medicine, University College Dublin, Ireland. Eran Zittan: Ellen and Pinchas Mamber Institute of Gastroenterology and Liver Diseases, IBD Unit, Emek Medical Center Afula Israel. Klaudia Farkas and Tamas Molnar: First Department of Medicine, University of Szeged Faculty of Medicine, Szeged, Hungary. Waqqas Afif: Division of Gastroenterology, McGill University Health Center, Montreal, Quebec, Canada. Fabiana Castiglione: Gastroenterology, Department of Clinical Medicine and Surgery, School of Medicine Federico II of Naples, Italy. Charles Lees: The Edinburgh IBD Unit, Western General Hospital, Edinburgh, UK. Manuel Barreiro-de Acosta: Unidad Ell, Servicio de Aparato Digestivo, Hospital Clínico Universitario de Santiago de Compostela, Santiago de Compostela, A Coruña, España. Triana Lobaton: Department of Gastroenterology, Ghent University Hospital, Ghent, Belgium. Glen Doherty: Centre for Colorectal Disease, St. Vincent's University Hospital and School of Medicine, University College Dublin, Ireland. Zeljko Krznaric: Gastroenterology Department, Rambam Health Care Campus, Haifa, Israel and Bruce Rappaport School of Medicine, TechnionIsrael Institute of Technology, Haifa, Israel. Marieke Pierik: Department of Internal Medicine, Division of Gastroenterology and Hepatology, Maastricht University Medical Center+, Maastricht, Netherlands. Frank Hoentjen: Department of Gastroenterology and Hepatology, Radboud University Medical Center, Nijmegen, the Netherlands. David Drobne: Department of Gastroenterology, University Medical Centre Ljubljana, Ljubljana, Slovenia and Medical Faculty, University of Ljubljana, Ljubljana, Slovenia. 